\title{
Unveiling DNA algorithms: satellite DNA sequences as iterable objects. A computational model.
}

\author{
Marco Regolini AudioLogic Milano (Italy)
}

\begin{abstract}
Every adult male of the little roundworm Caenorhabditis elegans is always and invariably comprised of exactly 1031 somatic cells, not one more, not one less; and so it is for the adult hermaphrodite (959 somatic cells); its intestine founder cell (the 'E' blastomere), if isolated and cultured, undergoes the same number of divisions as in the whole embryo (Robertson et al., 2014); the zygote of Drosophila melanogaster executes 13 cycles of asynchronous cell divisions without cellularization: how are these numbers counted? Artificial Intelligence (First and Second Order Logic, Knowledge graph Engineering) infers that, to perform precise stereotypical numbers of asynchronous cell divisions, a nucleic (genomic) counter is indispensable. Made up of tandemly repeated similar monomers, satellite DNA (satDNA) corresponds to iterable objects used in programming. The purpose of this article is to show how satDNA sequences can be iterated over to count a deterministic number of cell divisions: computational models (attached for free download) are introduced that handle DNA repeated sequences as iterable counters and simulate their use in cells through an epigenetic marker (cytosine methylation) as an iterator. SatDNA, because of its propensity to remodel its structure, can also operate as a strong accelerator in the evolution of complex organs and provides a basis to control interspecific variability of shapes.
\end{abstract}

\section{Introduction}

SatDNA plays an important role in the epigenetic machinery (Lacal and Ventura, 2018); many of its cytosines, (adenines in roundworms: Greer et al., 2015) are methylated by several enzymes (methylome: Ming et al., 2021): 'writers', DNA methyltransferases (DNMTs); 'readers', that interpret the significance of epigenetic modifications: cytosine methylation $(5 \mathrm{mC})$ often silences gene expression, hydroxymethylation ( $5 \mathrm{hmC}$ ) is associated with pluripotency (Won-Jun Lim, 2019); 'erasers', capable of removing methylations. Similar processes modify histones. Epigenetic modifications are copied and maintained during mitosis; deletions in DNMT genes are lethal in mouse embryos (Gao et al., 2020).

SatDNA shows a surprising self-remodeling skill because of its monomer similarity (Thakur et al,. 2021, Louzada et al., 2020): its rate of copying-errors is $10^{5}$ times higher than point mutations (Fondon and Garner, 2004); during evolution, replication slippages, non-disjunction, recombination, unequal crossing over, rolling-circle replications and multiple transpositions have generated diverse satDNA families, variously assembled in clusters of different textures and patterns: $\alpha$-satellite HORs (High Order Repeats) (Cacheux et al., 2016), long tandem arrays of RUs (Repeat Units) embedded in short interspersed tandem repeats (Ruiz-Ruano et al., 2016), Variable Number Tandem Repeats (VNTRs) (Wahls et al., 1990).

\section{Results}

Humans have about $10^{14}$ cells (Bianconi et al., 2013) 30,000 times their DNA base pairs, too many for each cell has its 'private' instructions; repetitive sequences, used as computer memories, can drive a large number of cell divisions through a small code, avoid 'infinite loops' (cancer) and achieve the desired target (parathyroids have fewer cells than thyroid). In the programs here presented, $n$ repeated satDNA monomers manage the emergence of $2^{\mathrm{n} / 2}$ cells: 8 monomers drive the generation of 16 cells; similarly, in D.mel. embryo the first 13 cycles of cell divisions can be counted by only 26 monomers $\left(2^{26 / 2}=8192\right.$ cells). Cellular arithmetical algorithms take advantage of a set of satDNA sequences of different lengths, similar to the rows of an abacus (a promoter, instead of a color, distinguishes each sequence; abacus rows have the 
same number of beads, satDNA sequences are made of variable quantities of head-to-tail disposed monomers); satDNA sequences are similar to electronic memory cells (of variable length) each one identified by its address; this set is limited, only some sequences have been selected and conserved in each species for their convenient length to realize particular tasks. A bookmarking mechanism is necessary that keeps track of each step and informs new cells about the moves already made: processive and ordered methylation of cytosine in a particular fixed position inside monomers is the supposed process that keeps the focus of the last step: new arisen cells read this bookmark-signal on inherited methylated DNA. Transcripts of DNA $\alpha$ satellite are indispensable for cell division (Bergman et al., 2012), cytosine methylation has a recognized role in silencing genes (Lim et al., 2019) and de novo methylation waves occur in mammal embryos (Lacal and Ventura, 2018). The main problem for mimicking this process is the 'privacy' of DNA: in programming, a list may be accessed, shared and changed by many functions or classes; on the contrary, a cell has its own ('deepcopied') DNA, can change it epigenetically and transmit it to its progeny, but other cells cannot access its DNA to know the changes operated on; after some cycles, cells possess satDNAs with different methylations: a shared epigenetically methylated DNA does not exist. To reproduce biological processes (and DNA algorithms) computer programs must utilize statements with a clear correspondence to known biological mechanisms: 'for...in', 'if', 'while', 'next', 'pop(0)' are the only statements used to implement the programs of this paper; 'while' there is a successive codon, 'pop(0)' 'and' process it: 'if it is 'not' a stop codon, check 'if a particular tRNA anticodon matches with it, 'else' try another tRNA. Classes, matrices, multidimensional arrays are indispensable in programming but no known biological action mechanism can execute recursive functions: implementing a binary tree (similar to cells) requires three parameters (cargo, left, right) per node: too many.

\section{The program 'Mitosis'}

A short repetitive DNA sequence, like CpG islands of pericentromeric $\alpha$-satellite, is used together with cytosine methylation as an epigenetic iterator: 'sat_DNA' is a list of eight 3'-5' palindromic monomers, nearly identical in composition, with terminal consensus motifs ( $3^{\prime}$ ATT5' and 3'TTA5'), necessary (Gao et al., 2020) as recognition sites for epigenetic 'readers'; the cytosine in the $5^{\text {th }}$ position is the bookmark; the first monomer possesses an adenine as the $6^{\text {th }}$ nucleotide (start sequence 'ATTCCA'), the last monomer has thymine in the $6^{\text {th }}$ position (stop sequence 'ATTCCT'). For simplicity the promoter, necessary for addressing the sequence, has been skipped and understood, only cytosines (not adenines as in roundworms) can be methylated and apoptosis has not been implemented. The process of methylation is reproduced as follows: in S phase, at the replication fork, histone-replication machinery, DNMTs, helicase, primase, PCNA, and topoisomerases (Lin-Yu et al., 2015) control the methylation process: (i) one cell ('first cell') inherits the methylated strand; the corresponding guanines on the opposite filament are marked by UHRF1 and histone H3, (Petryk et al., 2021), and, eventually, their matched cytosines are de novo methylated to equalize $5 \mathrm{mCs}$ in both sister cells; (ii) the 'first cell' recognizes the first not yet methylated monomer and immediately methylates it on its strand and also on the other one, belonging to its sister cell ('second cell') that will start reading from the next monomer.

A monomer is worked 'if' it is the first one, 'or if it follows a methylated monomer, 'and if its $5^{\text {th }}$ nucleotide is a not yet methylated cytosine $\left({ }^{\prime} C\right.$ '): after recognizing such a monomer, 'readers' are supposed to undergo an allosteric modification: information, likely a satRNA (Bergman et al., 2012), is carried to a control switch of the cell cycle; otherwise, if a methylated (' $M$ ') cytosine in the $5^{\text {th }}$ position silences the current monomer, the processive mechanism is activated and the next monomer is read, interpreted and executed; after processing a monomer, epigenetic complex methylates the $5^{\text {th }}$ nucleotide, unclasps and disengages from the DNA; so, a bookmarking signal $(5 \mathrm{mC})$ updates and keeps track of the counting process.

The $5^{\text {th }}$ nucleotide of the 'start' monomer is used to also state how the cell will divide: ' $C$ ' indicates a symmetric division into two 'twin sister' cells; ' $H$ ' ( $5 \mathrm{hmC}$ ) indicates an asymmetric division (stem cells): the cell will originate one 'unique daughter' differentiating cell: the function 'symm_mitosis' produces a symmetric cell-lineage while the function 'asymm_mitosis' reproduces stem cell divisions (Zion et al., 2020; 
Elabd et al., 2013). ' $A$ ' or ' $G$ ' in $4^{\text {th }}$ or $5^{\text {th }}$ position in any monomer is not recognized by 'readers': the process fails and stops.

A thymine ' $T$ ' as the $5^{\text {th }}$ nucleotide of any monomer means: 'the genetic code states that the present cell will no longer divide' (GO).

Running the program 'symm_mitosis', four symmetric cycles of cell divisions are executed. Different types of cell-lineages (selected during evolution), may be obtained by changing the $5^{\text {th }}$ ' $C$ ' nucleotide of one or more monomers with a ' $T$ '.

\section{The programs 'Morphogenesis' and 'Recursion\&Iteration'}

These programs deal with the evolution of complex organs, whose anatomy must evolve contemporaneously: perfect eyes without a perfect brain are useless. 'Morphogenesis' and

'Recursion\&Iteration' show how satDNA can be the key to fast the evolution of complex organisms. Virusmediated DNA transfer accelerates the evolution of unicellular, fast-developing, living beings: what is the 'accelerator' in big organisms? Random 'single-nucleotide' errors in copying DNA are the most frequent agents in evolution (Katsonis et al., 2014) but they are too slow for big organisms and their complex organs (slow growth, long time from birth to fertility, transmissible mutations only in germinal cells and sophisticated organ architectures). In the program 'Morphogenesis' the call of the function 'koch' generates an impressive variation of shape (Fig. 1 and 2) when it is written with a $6(0110 \underline{010})$ in place of a 2 (0110110) ( $k$ och $(l, r, 2,5 / \operatorname{koch}(l, r, 6,5))$ : only one bit of difference; this simple trivial 'point mutation' would be a powerful 'genetic lever', capable of performing large phenotypic changes using small (and then likely) genomic variations. Is it possible in living beings? More than likely, yes: satDNA 'self-remodeling' propensity is the key that can bridge the gap between micro and macroevolution. 'Morphogenesis' uses the Koch curve: fractals (and Fibonacci sequences) are fascinating, describe real biological geometries (pineapple, sunflower, cabbage, phyllotaxis) but no known biological mechanism executes recursive functions: recursion can be implemented by the repetition of 'for...in' cycles, much more similar to biological processive processes (Fig.3). 'Recursion\&Iteration' indentations reproduce successive evolutionary DNA-duplications (easy evolutionary events) capable of realizing fractals and Fibonacci sequences: higher-order clusters, as a consequence of satDNA self-remodeling skill, are implemented like: $1(X) \rightarrow 2\left(X_{x} X\right) \rightarrow 3\left[\left(X_{x} X\right)_{x}\left(X_{x} X\right)\right] \rightarrow 4\left\{\left[\left(X_{x} X\right)_{x}\left(X_{x} X\right)\right]_{x}\left[\left(X_{x} X\right)_{x}\left(X_{x} X\right)\right]\right\} \ldots$ resumed as: $2=1$ 1; 3=2_2; 4=3_3. Cluster duplications of satDNA constitute a credible evolutional mechanism for fast and large variations of shapes.

\section{The program 'Iter'}

Controlled iteration of simple functions can realize basic (bilaterally symmetric) biological shapes. 'Iter' uses two sat_DNA sequences: a counter and a sequence of angle codes (strings of five nucleotides similar to amino-acid-codons): the first three nucleotides are a consensus sequence (ATT), the last two are the code for a multiple of the centriolar $40^{\circ}$ degree: the redundancy of the code for 9 items $\left(360^{\circ} / 40^{\circ}\right.$; dispositions with repetitions of 2 objects out of 4 is $4^{2}$, more than 9) has been taken into account. To realize bilaterally symmetric structures (almost all animals are Bilateria), casual implementations of two different programs is an extremely unlikely evolutionary event: casual errors during the process of DNA replication are the unique evolutionary tools, therefore two different 'symmetric' programs, in every species and for every organ, should have been produced by chance: impossible. Two executors, one 'righthanded' and one 'lefthanded, follow the same instructions but build two symmetric objects with a common midline. The difference is inside the 'executor' (the 'Python Turtles') and not in the program: this is much more evolutionarily likely, because it is realized once and for all in a quite easy way: in 'Morphogenesis' and 'Iter' a unique line of code generates two symmetric executors. 'Iter' reproduces a mechanism that translates genetic geometric instructions into real spatial locations: after transcribing the sequence 'sat_DNA_degree' into a 'sat_RNA', this is read step by step ('sat_RNA'.pop(O)), information is transferred to the variable ' $x$ ' (similar to a tRNA) that drives the cell to chois the correspondent triplet on the centrosome and perform geometric tasks (Regolini, 2019). 'Iter' realizes a circular structure using a satDNA sequence to count the number of repetitions of elementary 
functions; it shows that satDNA sequences are a strong base for evolutive changes: simple (and then likely) mutations in the sequences 'sat_DNA_degrees' and 'sat_DNA_count' are capable of generating impressive and large changes of shape.

Combining Mitosis' and 'Iter' in the program 'Bilateral mitosis', two satDNA sequences (a counter and an angle-coding list) are used: two functions of only one line set up two symmetric executors and corresponding cell-lineages, satDNA of each cell, and the symmetric spatial disposition of generated cells are printed.

\section{Discussion}

\section{SatDNA sequences to count}

Gene diversity (very little) between monkeys, apes and humans cannot explain their (very large) neuroanatomy differences; the real disparity is not inside the coding DNA but in the so-called 'dark matter' (satellitome and repeatome) (Ahmad et al., 2020): satDNA diversity in quantity, and, above all, in quality (cluster organization) can control (count) the relevant difference in the number of neuroblast divisions. DNA-algorithm implemented for counting deterministic cell divisions is simple: (i) the epigenetic complexes of a 'founder' cell are addressed (as memory cells are accessed in computers) to the promoter of the satDNA sequence responsible for counting the process in execution; (ii) the bookmark $(5 \mathrm{mC})$ is recognized, the next not silenced monomer is read and, in turn, bookmarked; (iii) information is sent to divide (or not); (iv) the epigenetic complexes of newly born cells recognize the bookmark left on inherited DNA by their mother cell, and the process restarts.

Query: is this rationale correct? Satellite DNAs are made up of tandemly repeated similar nucleotide sequences, each one supposed to be univocally identifiable by its promoter: are they iterable objects capable of counting a deterministic number of asynchronous cell divisions? 'Knowledge Graph Engineering' confirms this assertion. Biochemical and biophysical concepts, many known biological functions and processes ('true' sentences for a knowledge base) have been given to the machine for testing the previous assertion: by Al First and Second Order Logic, processes like the segmentation of $D$. mel. zygote, are impossible in the absence of a nucleic, genetic, linearly processive, counting mechanism.

To test the correctness of the program 'Mitosis' different deep learning methods have been proved (stochastic gradient descent, random forest); Artificial Neural Networks (two hidden layers Keras/TensorFlow) have been used; algorithms similar to those of 'Cellular Automata' and 'Game of Life' have been utilized to prepare short lists (training data) to fit the machine: instead of considering lists of 'neighborhood', 'lineage' lists for progeny (from parent to child) have been built, made up of chains of '0's ('cell cycle exit') and '1's ('divide again') to establish the fate of each descendant of the founder cell; lists were of different lengths for a different number of cell cycles and there was no identity between ' 0 's and ' 1 's of a list and those ( $1=$ existing_cell, $0=$ not_existing-cell) of the final set of its corresponding offspring: the list $[1,1,1,0,1,1,1,1]$ corresponds to $[1,1,0,0,0,0,1,1,0,0,0,0,0,0,1,1]$ lineage.

2D printed lineages do not draw real 3D geometric distributions of cells (Giurumescu and Chisholm, 2011): numbers or letters identify a particular cell in that for each one it is possible to trace progenitors and offspring, without any reference to their spatial disposition: many different geometric results are possible for the same lineage.

\section{SatDNA sequences and evolution}

SatDNAs, as seen, can operate as accelerators in the evolution of complex organs. 'Recursion\&lteration' draws curves, per se insignificant, but is quite interesting for the code with ordered duplications/repetitions; transposable elements are often interspersed in satDNA (Sander Lower et al., 2018): in the program, nested loops resemble satDNA cluster organization (VNTRs, RU and HORs) built during evolution by insertions of multiple extra-copies, massive transpositions of satDNA sequences prone to self-remodeling. Not only: probabilism is often invoked to explain intraspecific shape variations ('probabilism' is a misleading word: 'cells do not play dice' a misquoted famous aphorism says...); unlimited randomness is dangerous, chaotic growth must be prevented, therefore the basic planes of growth are strictly deterministic (a cat is a cat and a mouse is a mouse), whereas terminal stages permit some 
individual differences (each individual has its peculiarities); so, variability is confined to a limited range in the last developmental stages for a controlled individual diversity (human female/male height distributions show a standard deviation of $\approx 3$ inches, $\approx 5 \%$ of the mean value): in this context, variable cluster organisation of satDNA (VNTRs above all) constitute an ideal molecular base for intraspecific variability (not by chance VNTRs are named DNA 'fingerprints'). Hormones, signaling molecules and morphogens can operate on these clusters of satDNA, stopping or restarting methylation cycles.

\section{Bilateral symmetry}

'Morphogenesis' and 'Iter' design symmetrical structures using two 'symmetric' executors (Turtles) different for only one byte of code ('r.left(-90)'//'I.left(90)'): this is quite intriguing to reproduce a credible evolutionary event for bilateral symmetry emergence. Two symmetric compasses (East and West swapped) run symmetrical pathways under the same instructions. In Bilateria a sagittal plane and a midline are evident in unpaired organs too: cranium, backbone, pelvis, tongue); the left/right (and inter-individual) precision of D.mel. wings are specified with the astonishing spatial precision of one cell width (Abouchar et al., 2014). Bilateria have a centrosome made up of two orthogonal centrioles, attached to the cell membrane by an aster of microtubules and responsible for the mitotic spindle.

'Morphogenesis' and 'Iter' (Fig. 2 and 4) realize bilateral symmetry using a unique program for both left and right sides: only one byte of code $\left({ }^{\prime}-{ }^{\prime}\right)$ prepares two, left- and right-handed, executors that follow the same instructions but produce symmetric results. What is the invertible 'cellular compass' (feasible with an invertible medial-lateral axis)? A suggestion comes from chemistry and mineralogy: enantiomorph molecules generate enantiomorph crystals, right- and left-handed, as quartz.

Centrioles show a typical 9-fold symmetry founded on SAS-6 protein capable of building regular polymers of nine sides, connected at $140^{\circ}$, facing an internal angle of $40^{\circ}$ (Gopalakrishnan et al., 2010). Rotational asymmetry of centrioles and cilia basal bodies have been confirmed by researches on Protists: their nine triplets are not equivalent and assembled in a defined and sorted sequence; circumferential polarity has been described also in mammals (Beisson and Jerka-Dziasdosz, 1999, Geimer and Melkonian, 2004, Pearson and Winey, 2009, Bengueddach et al., 2017; Regolini, 2019). Live images by Ferreira et al. (2019) show that in the zebrafish Kupffer's vesicle (the left-right organizer, homologous of the chick node) cilia are asymmetrically orientated in the right and left sides: primary cilia (and their centriole/basal bodies) possess a real chiral structure, not a simply chiral rotation. In 'Morphogenesis' printed points represent new cells: 'forward' reproduces the process of cell divisions without changing the orientation of the division plane, 'left $\left(\theta^{\circ}\right)$ ' and 'right $\left(\theta^{\circ}\right)$ ' simulate a change of direction; two bilaterally symmetric complex structures are generated (the midline is intentionally crossed in three positions, like pyramidal decussation or optic chiasma in many vertebrates).

In 'Iter' it is shown, step by step, how a list of angles (a sequence of genetic codes relative to angles) can drive the disposition of the mitotic spindle: forecasted and coded genetic instructions locate new daughter cells, division after division, in the desired positions relative to the mother cell.

It is likely that, from a reverse transposition (Alliegro et al., 2006; 2008; Chichinadze, 2013; Regolini 2016), a circular enantiomorph RNA has emerged, capable of reversing the rotational polarity of the centriole and generating two symmetric enantiomorphs, right- and left-handed, centrioles: built in the very first blastomeres, they can generate (as quartz) left and right symmetric organs following a unique developmental code. Centrosomes as geometrical organizers (Regolini, 2019) and enantiomorph centrioles may realize these tasks: Al tests (First Order Logic) find 'not false' this assertion.

\section{References}

Abouchar L., Petkova M.D., Steinhardt C.R. and Gregor T. 2014 Fly wing vein patterns have spatial reproducibility of a single cell. J. R. Soc. Interface:1120140443

Ahmad S.F. Singchat W. et al. 2020 Dark matter of primate genomes: satellite DNA repeats and their evolutionary dynamics. Cells (09): 02714 
Alliegro M.C., Alliegro M.A. 2008 Centrosomal RNA correlates with intron-poor nuclear genes in Spisula oocytes. Proc. Natl. Acad. Sci. USA 105: 6993-6997.

Alliegro M.C., Alliegro M.A., Palazzo R.E. 2006 Centrosome-associated RNA in surf clam oocytes. Proc. Natl. Acad. Sci. USA 103: 9034-9038

Beisson J., Jerka-Dziadosz M. 1999 Polarities of the centriolar structure: morphoge-netic consequences. Biol. Cell 91: 367-378

Bengueddach, H., Lemullois, M., Aubusson-Fleury, A. et al. 2017Basal body positioning and anchoring in the multiciliated cell Paramecium tetraurelia: roles of OFD1 and VFL3. Cilia 6, 6

Bergmann J.H., Jakubsche J.Net al. 2013 Epigenetic engineering: histone H3K9 acetylation is compatible with kinetochore structure and function. J Cell Sci 125 (2): 411-421.

Bianconi E., Piovesan A., Facchin F. et al. 2013 An estimation of the number of cells in the human body. Annals of Human Biology (40): 6

Cacheux, L., Ponger, L., Gerbault-Seureau, M. et al. 2016 Diversity and distribution of alpha satellite DNA in the genome of an Old World monkey: Cercopithecus solatus . BMC Genomics (17): 916

Chichinadze K. 2013 RNA in centrosomes: structure and possible functions. Protoplasma 250:397-405

Elabd C.., Cousin W, Chen R.Y., et al. 2013 DNA methyltransferase-3-dependent nonrandom template segregation in differentiating embryonic stem cells. J Cell Biol. 203(1):73-85

Ferreira R.R., Vilfan A., Pakula G., Supatto W., Vermot J. 2018 Chiral cilia orientation in the left-right organizer. Cell Rep. 25(8)

Fondon, J. W. and Garner, H. R. 2004 Molecular origins of rapid and continuous morphological evolution. Proceedings of the National Academy of Sciences (101): 18058-18063

Gao, L., Emperle M., Guo Y. et al. 2020 Comprehensive structure-function characterization of DNMT3B and DNMT3A reveals distinctive de novo DNA methylation mechanisms. Nature Comm. (11): 3355

Geimer S., Melkonian M. 2004 The ultrastructure of the Chlamydomonas reinhardtii basal apparatus: identification of an early marker of radial asymmetry inherent in the basal body. J. Cell Sci. 117: 2663-2674

Giurumescu, C. A., Chisholm, A. D. 2011. Cell identification and cell lineage analysis. Methods in cell biology, 106: 325-341

Gopalakrishnan J., Guichard P., Smith A.H., et al. 2010 Self-assembling SAS-6 multimer is a core centriole building block. J Biol Chem. 285(12):8759-7

Greer E. L., Blanco, M. A. et al., 2015 DNA Methylation on N6-Adenine in C. elegans. Cell 161(4): 868-878

Katsonis P., Koire A., et al. 2014 Single nucleotide variations: biological impact and theoretical interpretation. Protein Sci. 23(12):1650-66. 
Lacal I. and Ventura R. 2018 Epigenetic Inheritance: Concepts, Mechanisms and Perspectives. Front. Mol. Neurosci. (11): 292

Lim W.J., Kim K.H., Kim J.Y., Jeong S., Kim N. 2019 Identification of DNA-Methylated CpG Islands Associated With Gene Silencing in the Adult Body Tissues of the Ogye Chicken Using RNA-Seq and Reduced Representation Bisulfite Sequencing. Front Genet. 16(10): 346.

Louzada S., Lopes M., et al. 2020 Decoding the Role of Satellite DNA in Genome Architecture and Plasticity-An Evolutionary and Clinical Affair Genes 2020, 11(1), 72

Ming X., Zhu B., Li Y. 2021 Mitotic inhertitance of DNA methylation: morethan just copy and paste. J. Genet. Genomics 48(1): 1-13

Pearson C.G., Winey M. 2009 Basal body assembly in ciliates: the power of num-bers. Traffic 10: 461-471

Petryk N., Bultmann S., Bartke T, Defossez P. 2021 Staying true to yourself: mechanisms of DNA methylation maintenance in mammals. Nucleic Acids Research, 49

(6):3020-3032

Regolini, M. 2013. Centrosome: is it a geometric, noise resistant, 3D interface that translates morphogenetic signals into precise locations in the cell?. Italian Journal of Anatomy and Embryology, 118(1), 19-66.

Regolini, M. 2019. Centriole enantiomerism: unexpected information from mice and fish. Italian Journal of Anatomy and Embryology, 123(3), 232-240.

Regolini M. 2015 Centrosome RNA: A Molecular Basis for Non-Equivalence of Triplets in Centrioles and Centrosomes. Mol Biol 4: 125

Regolini M. 2019 The Centrosome as a Geometry Organizer. Results Probl Cell Differ. Springer Nature editor 67:253-276.

Robertson S.M., Medina J., Lin R. 2014 Uncoupling Different Characteristics of the C. elegans E Lineage from Differentiation of Intestinal Markers. PLoS ONE 9:9

Ruiz-Ruano, F. J., López-León, M. D., Cabrero, J., \& Camacho, J. 2016 High-throughput analysis of the satellitome illuminates satellite DNA evolution. Scientific reports, (6): 28333.

Thakur J., Packiaraj J., Henikoff S. 2021 Sequence, Chromatin and Evolution of Satellite DNA. Int. J. Mol. Sci. 2021, 22(9), 4309

Wahls W. P., Wallace L.J., Moore P. D.1990 Hypervariable minisatellite DNA is a hotspot for homologous recombination in human cells. Cell 60(1): 95-103.

Zion E.H., Chandrasekhara C., Chen X. 2020 Asymmetric inheritance of epigenetic states in asymmetrically dividing stem cells. Curr Opin Cell Biol. 67:27-36 


\section{5}

Fig.1 Graphic of the function 'koch' called with 'koch(l,r, 2,5)'

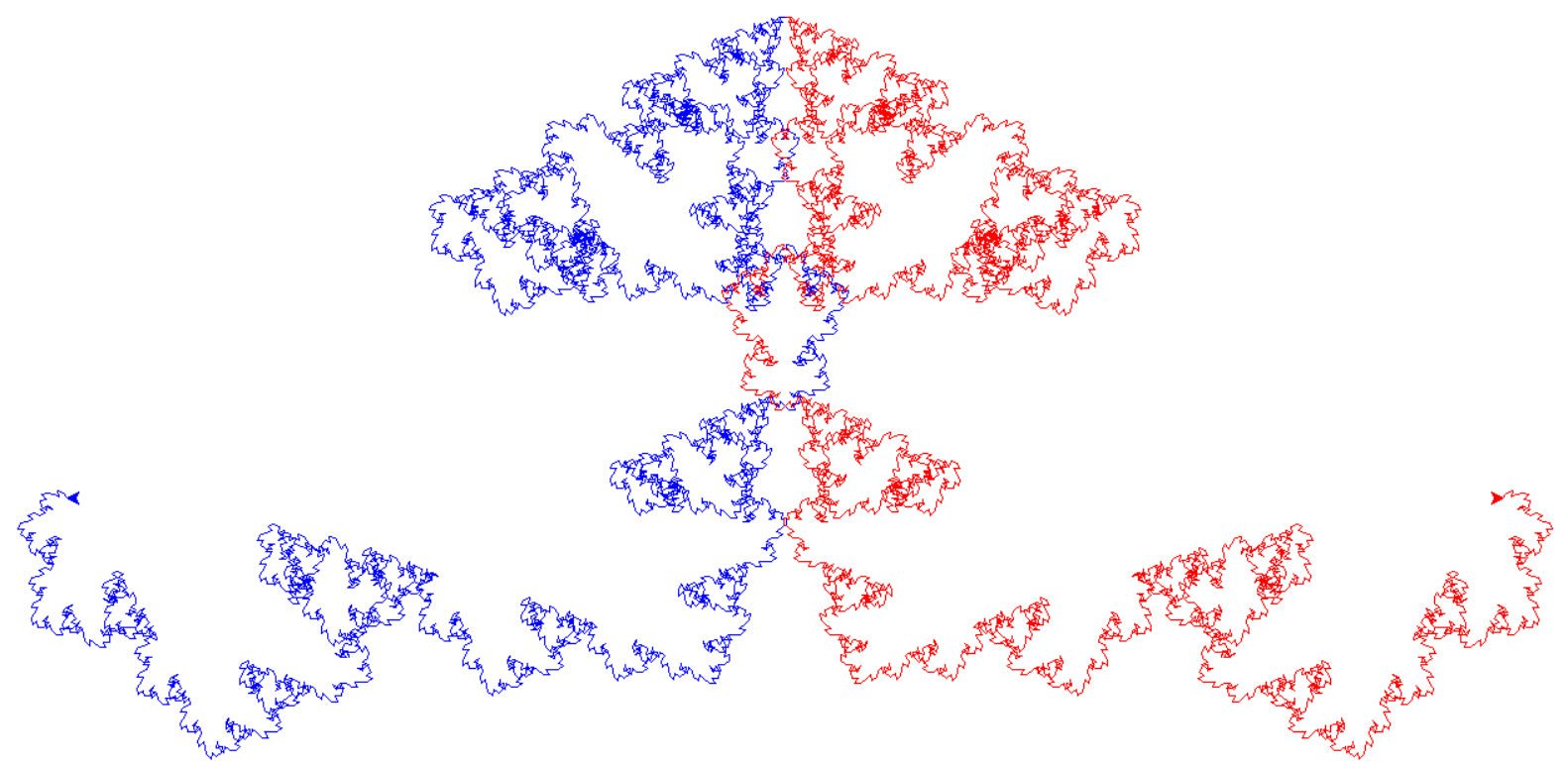

Fig. 2 Graphic of the function 'koch' called with 'koch $(1, r, 6,5)$ '; $k$ och $(1, r, 6,5)$ instead of koch $(1, r, 2,5)$ generates an impressive variation of shape. Note that the difference between the codes of ' 6 ' and ' 2 ' consists in only one bit (“0110ㅁ10' instead of '0110 110 '): a 'single nucleotide' error in the $5^{\text {th }}$ digit. 


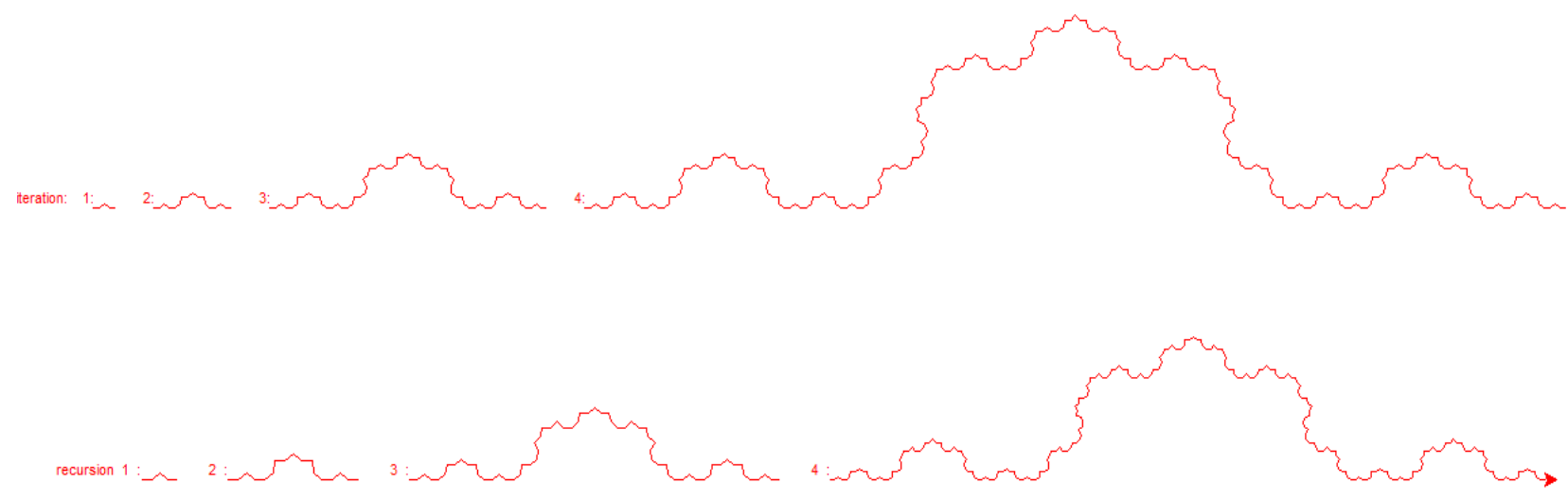

Fig. 3 


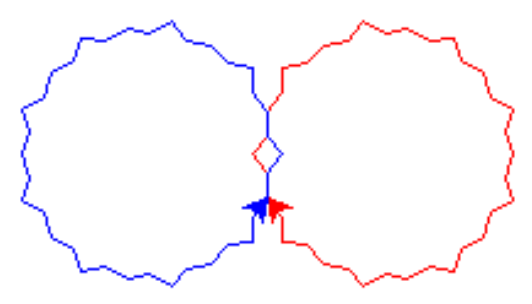

Fig. 4 